American Journal of Applied Sciences 6 (5): 970-973, 2009

ISSN 1546-9239

(C) 2009 Science Publications

\title{
Electricity Load Forecasting based on Framelet Neural Network Technique
}

\author{
Mohammed K. Abd \\ Department of Electrical and Electronic Engineering, University of Technology, Baghdad, Iraq
}

\begin{abstract}
Load forecasting is very essential to the operation of electricity companies. It enhances the energy-efficient and reliable operation of a power system. This study shows Electricity Load Forecasting modeling based on Framelet Neural Network Technique (FNN) for Baghdad City. Framelet technique is implemented to the time series data, decomposing the data into number of Framelet coefficient signals. The decomposed signals are then fed into neural network for training. To obtain the predict forecast, the outputs from the neural network are recombined using the same Framelet technique. The simulation results showed that the model was capable of producing a reasonable forecasting accuracy in short term load forecast.
\end{abstract}

Key words: Load forecasting, framelet, neural network, series time data.

\section{INTRODUCTION}

Many power systems not only are being pushed to their limits to meet their customers' demands, but also spend a lot of resources in their operation scheduling. System security is becoming an ever more important issue in modern electrical power systems. It is known for all that electrical energy cannot be stored efficiently, due to this fact that the electrical load could be controlled by utilities only to a very small extent, the forecasting of load demand data forms an important component in planning generation schedules in a power system.

The short term load forecast is to predict future electricity demands based on historical data and other information such as temperature. Time series forecast is traditionally based on linear models e.g., Autoregressive Moving Average (ARMA) models ${ }^{[2}$ ], have been used for Load Forecasting ${ }^{[4]}$. These models are straight for implementation but fail to give satisfactory results when dealing with nonlinear, non-stationary time series. The solution to that is Neural Network as supervised models have been used to deal with the nonlinearity and non-stationary in electricity load prediction and have produced good and satisfactory results $^{[1,3,5]}$, for their approximation ability for nonlinear mapping and generalization. However, it suffers from the problem of obtaining monolithic global models for a time series. This introduced the multi-resolution decomposition techniques such as Framelet transform approach. Framelet transforms provide a useful decomposition of the time series, in terms of both time and frequency. They have been used effectively for image compression, noise removal, object detection and large-scale structure analysis, among other applications $^{[5]}$.

For this study, combining the learning capabilities of neural networks and time series techniques and using Framelet decomposition technique to explore more details of the time series signals. A multi-layer perception neural network is applied to each decomposed series to predict the time series. We will study neural network models combined with Framelet transformed data, and show how useful information can be captured on various time scales.

Framelet technique: Framelet are very similar to wavelets but have some important differences. In particular, whereas wavelets have an associated scaling function $\Phi(\mathrm{t})$ and wavelet function $\psi(\mathrm{t})$, framelets have one scaling function $\Phi(\mathrm{t})$ and two wavelet functions $\psi 1(\mathrm{t})$ and $\psi 2(\mathrm{t})$.

The scaling function $\Phi(\mathrm{t})$ and the wavelets $\psi 1(\mathrm{t})$ and $\psi 2(\mathrm{t})$ are defined through these equations by the low-pass (scaling) filter $\mathrm{h}_{0}(\mathrm{n})$ and the two high-pass (wavelet) filters $h_{1}(n)$ and $h_{2}(n)$. Let $\Phi_{k}(t)=\Phi(t-n)$ and:

$\psi_{i, j, k}(t)=\psi_{i}\left(2^{j} t-k\right)$ for $\quad i=1,2$

Any function $\mathrm{f}(\mathrm{t})$ could be written as a series expansion in terms of the scaling function and wavelets by $^{[6]}$ :

$\mathrm{f}(\mathrm{t})=\sum_{\mathrm{k}=-\infty}^{\infty} \mathrm{c}(\mathrm{k}) \varphi_{\mathrm{k}}(\mathrm{t})+\sum_{\mathrm{j}=0}^{\infty} \sum_{\mathrm{k}=-\infty}^{\infty} \mathrm{d}_{1}(\mathrm{j}, \mathrm{k}) \psi_{1, \mathrm{j}, \mathrm{k}}(\mathrm{t})+\mathrm{d}_{2}(\mathrm{j}, \mathrm{k}) \psi_{2, \mathrm{j}, \mathrm{k}}(\mathrm{t})$

Where:

$c(k)=\int f(t) \varphi_{k}(t) d t$ 


$$
d_{i}(j, k)=\int f(t) \psi_{i, j, k}(t) d t \quad i=1,2
$$

In this expansion, the first summation gives a function that is a low resolution or coarse approximation of $\mathrm{f}(\mathrm{t})$ at scale $\mathrm{j}=0$. For each increasing $j$ in the second summation, a higher or finer resolution function is added, which adds increasing details.

A Framelet base is employed in the hidden layer of FNN rather than a sigmoid function, which discriminates it from general back propagation neural networks ${ }^{[7,8]}$.

The training algorithm of FNN is elucidated as following steps:

Step 1: Data acquisition: The output response voltage signals of test point are sampled in terms of the SY running under different input step voltage. Then optimal features for training neural networks are obtained by wavelet decomposing coefficients and normalization

Step 2: Select the parameters of wavelet neural network: The dimension of fault feature vectors and the circuit fault pattern determines the number of input node and output node. The output number is equal to the number of fault classes, while input number is equal to the dimension of feature vectors. The number of neurons on hidden layer is set greater or equal to $\sqrt{\mathrm{M}+\mathrm{N}}+\mathrm{a}$, where $\mathrm{M}$ and $\mathrm{N}$ is the node number of input layer and output layer and is set $1 \sim 10$

Step 3: Training of wavelet neural network: The different input step voltage vectors, as input vectors of training pattern, are used to train the wavelet neural network. The output vectors reflect the states of SY. The gradient descent algorithm is employed to minimize the error function so as to adjust the weights of network. The error function is:

$E=\frac{1}{2} \sum_{p=1}^{p} \sum_{j=1}^{j}\left(y_{j}^{p}(t)-y_{j}(t)\right)$

where $\mathrm{P}$ is the total number of training patterns ${ }^{*}{ }_{j}^{*}(t)$ and $y_{j}^{p}(t)$ are the desired and real output associated with the jth feature for the pth neuron.

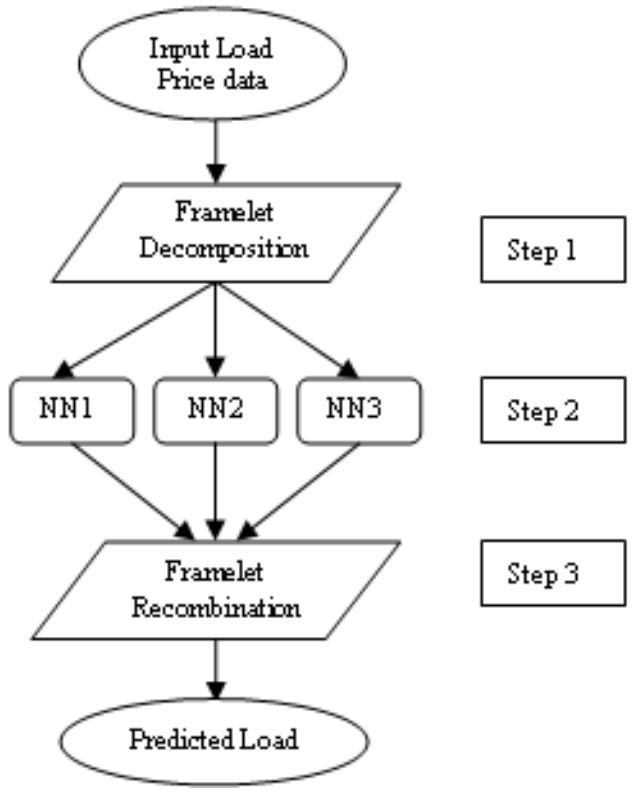

Fig. 1: Flowchart of the forecast based on FNN

\section{MATERIALS AND METHODS}

Electricity load forecasting based on FNN: The block diagram of the forecasting model principle based on framelet neural network is given in Fig. 1. The data used in the model to train neural networks are historical electricity load and pricing data. As for the data process tool Framelet Technique is used in the model.

The model consists of three stages:

Step 1: Data Pre-processing

Step 2: Data Prediction

Step 3: Data Post-processing

The preliminary forecast model has three main stages. This is illustrated in flowchart below;

Model validation: The model is evaluated based on it prediction errors. A successful model would give an accurate time-series forecast. The performance of the model is hence measured using the Mean Absolute Percentage Error (MAPE) which is defined as:

Absolute Percentage Error (APE), which is defined as:

$\operatorname{APE}(\%)=\frac{\mid \text { Actual(i) }- \text { Forecast }(\mathrm{i}) \mid}{\operatorname{Actual}(\mathrm{i})} * 100$

Mean Absolute Percentage Error (MAPE), which is defined as follows: 
$\operatorname{MAPE}(\%)=\frac{1}{\mathrm{x}} \sum_{\mathrm{i}=1}^{\mathrm{x}} \frac{\mid \operatorname{Actual}(\mathrm{i})-\text { Forecast (i) } \mid}{\text { Actual(i) }} * 100$

Where:

$\mathrm{X}=$ The total number of hours predicted

Actual(i) = The actual load for the hour i,

Forecast $(\mathrm{i})=$ The predicted load for the hour $\mathrm{i}$.

\section{RESULTS AND DISCUSSION}

Model simulation result: The proposed model is tested with two sets of historical data containing the electricity load and price data of Baghdad City as shown in Fig. 2, on a half-hourly basis, 1-7 June 2004.

Price is only used with load to form a particular time-series pattern as the inputs for training the neural networks, the model output was only the predicated electricity load and tested with a number of different values of hidden neurons, however no significant changes are observed with the predicted results. Therefore for this study, the number of hidden neurons is set to two.

The model consists of three steps:

Step one: Demand and price historical time-series data of Baghdad City are decomposed at resolution level 2 as shown in Fig. 3 (A and B) respectively .

Step two: As depicted in forecast model, three neural networks were created for the forecast model-one for the approximation series and one for each of the two Framelet coefficient series. The neural networks were trained with 1 day (48 points), 2 days (98 points),

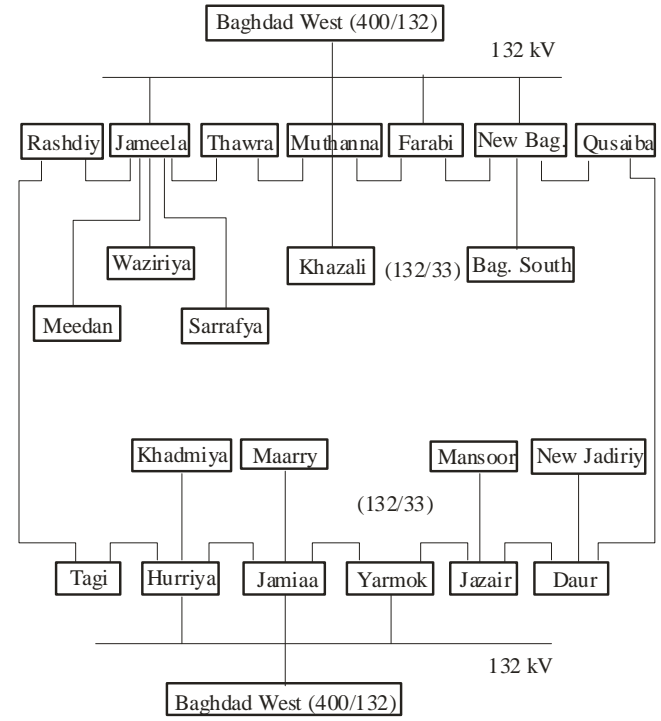

Fig. 2: Distribution network structure of Baghdad city
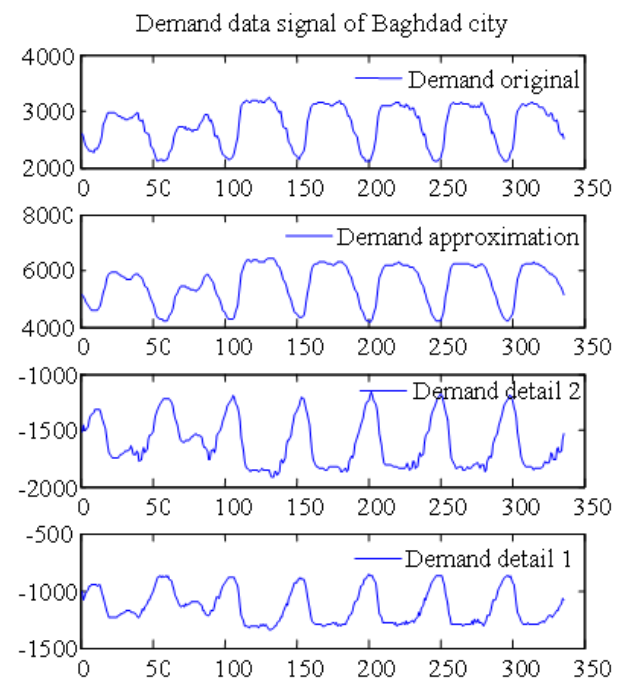

(a)
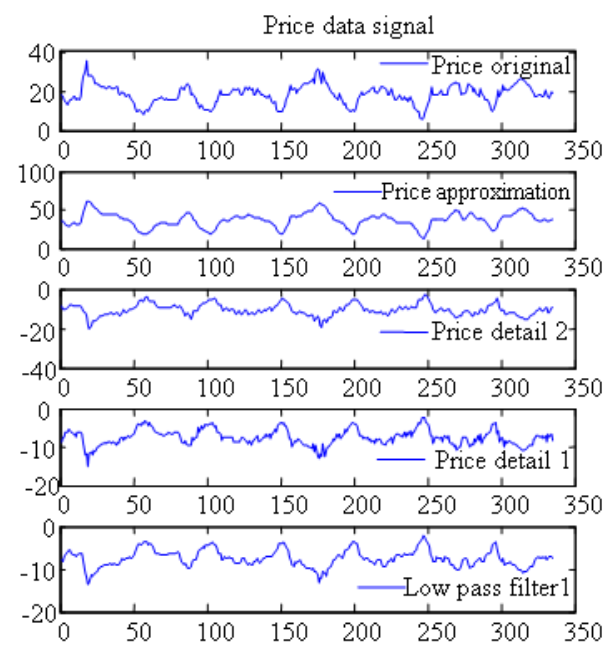

(b)

Fig. 3: Decomposition of Baghdad city (a)Demand series data(b) Price series data

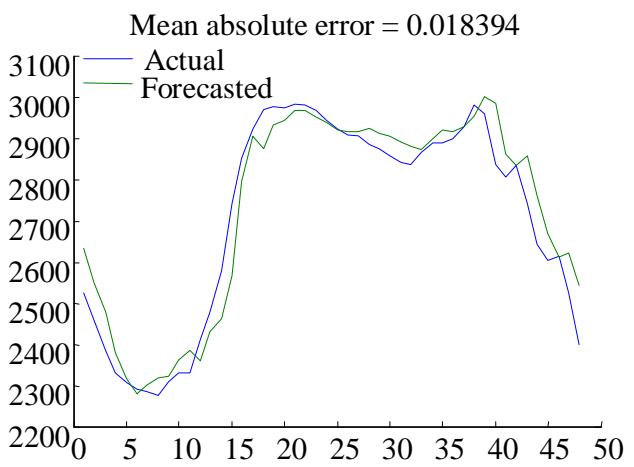

Fig. 4: Load Forecasted for 1 day (48 points) 


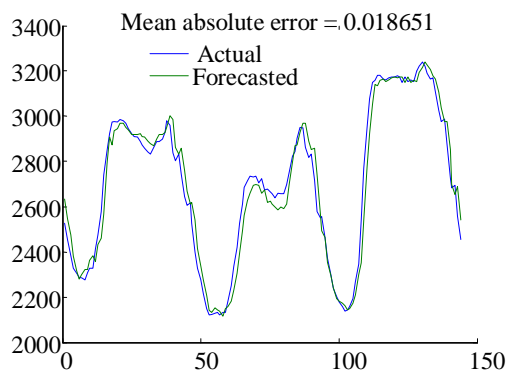

Fig. 5: Load forecasted for 3 days (144 points)

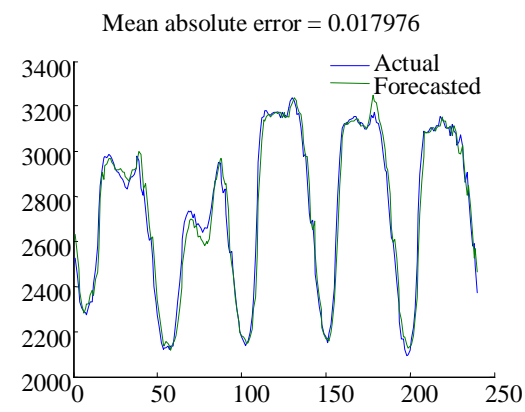

Fig. 6: Load forecasted for 5 days (240 points)

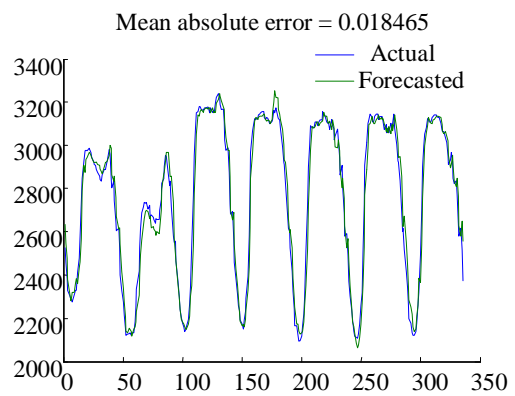

Fig. 7: Load forecasted for 7 days (336 points)

Table 1: Summary of MAPEs

\begin{tabular}{ll}
\hline No. of forecasted points & Mean absolute error $(\%)$ \\
\hline 48 (1 days) & 0.018394 \\
96 ( days) & 0.019877 \\
144 ( 3 days $)$ & 0.018651 \\
192 (4 days) & 0.018105 \\
240 (5 days) & 0.017976 \\
288 ( 6 days $)$ & 0.018313 \\
336 ( days $)$ & 0.018465 \\
\hline
\end{tabular}

3 days (144 points), 4 days (192 points), 5 days (240 points), 6 days (288 points) and 7 days (336 points) of combined load and price data for fifty cycles.

Step three: The performance of the forecast model was evaluated and the results are as shown in Fig.4-7.

Model validation: The performance of the proposed model is measured by the MAPE using Eq. 6. These
MAPEs were recorded in Table 1 . The MAPE calculated is less than $0.019877 \%$ of Baghdad city.

\section{CONCLUSION}

This study proposed a LFFNN model with a high forecasting accuracy. The FNN has been successfully implemented in the model. The results obtained in this work confirm the applicability as well as the efficiency of neural networks in short-term load forecasting. The implementation of FNN has reasonably enhanced the learning capability of the NNs in the model, thus minimizing their training frequencies as shown in the simulations. The neural network was able to determine the nonlinear relationship that exists between the historical load data supplied to it during the training phase and on that basis, and make a prediction of what the load would be in the next and the use of FNN (as the data processing tool) for the proposed model have been a success.

\section{REFERENCES}

1. Chow, T.W.S. and C.T. Leung, 1996. Neural networks based short term load forecasting using weather compensation. IEEE Trans. Power Syst., 11: 1736-1742. DOI: $10.1109 / 59.544636$

2. Francisco, N.J. and C. Javier, et al., 2002. Forecasting next-day electricity prices by times series models. IEEE Trans. Power Syst., 17: 342-348. DOI: 10.1109/TPWRS.2002.1007902

3. Hipert, H.B. and C.E. Pedreira, et al., 2001. Neural-networks for short term load forecasting: A review and evaluation. IEEE Trans. Power Sys., 16: 44-55. DOI: $10.1109 / 59.910780$

4. Moghram, I. and R. Saifur, 1989. Analysis and evaluation of five short-term load forecasting techniques. IEEE Trans. Power Syst., 4: 1484-1491. DOI: $10.1109 / 59.41700$

5. Khtanzad, A., R. Afkhami-Rohani and D.J. Maratukulam, 1998. ANN STLF artificial neural network short-term load forecaster-generation three. IEEE Trans. Power Syst., 13: 1423-1422.

6. Selesnick, I.W., 2001. Smooth wavelet tight frames with zero moments. Applied Comput. Harmonic Anal. 10: 163-181. DOI: 10.1006/acha.2000.0332

7. Kingsbury, N.G., 1998. The dual-tree complex wavelet transform: A new technique for shift invariance and directional filters. Proceedings of the 8th IEEE DSP Workshop' 98, Utah, pp: 319-322. http://citeseerx.ist.psu.edu/viewdoc/summary?doi= 10.1.1.51.1213

8. Al-Taai, H.N., 2008. A Novel fast computing framelet coefficients method. Am. J. Appli. Sci., 5: 1522-1527. http://www.scipub.org/fulltext/ajas/ajas51115221527.pdf 